\title{
FPGA-BASED ACCELERATION OF THE COMPUTATIONS INVOLVED IN TRANSCRANIAL MAGNETIC STIMULATION
}

\section{Octavian Creț, Ionuț Trestian, Radu Tudoran, Laura Creț, Lucia Văcariu}

\author{
Computer Science and Electrotechnics \\ Department \\ Technical University of Cluj-Napoca \\ 26 Barițiu street, Cluj-Napoca, Romania \\ email: Octavian.Cret@cs.utcluj.ro
}

\section{Florent de Dinechin}

\author{
LIP, Ecole Normale Supérieure de Lyon \\ UMR CNRS/INRIA/ENS-Lyon/Université \\ Claude Bernard Lyon 146 allée d'Italie, \\ 69364 Lyon cedex 07, France \\ email: Florent.de.Dinechin@ens-lyon.org
}

\begin{abstract}
In the last years the interest for magnetic stimulation of the human nervous tissue has increased, because this technique has proved its utility and applicability both as a diagnostic and as a treatment instrument. Research in this domain is aimed at eliminating some disadvantages of the technique: the lack of focalization of the stimulated human body region and the reduced efficiency of the energetic transfer from the stimulating coil to the tissue. Designing better stimulation coils is so far a trial-and-error process, relying on very compute-intensive simulations. In software, such a simulation has a very high running time (several hours for complicated geometries of the coils). This paper proposes and demonstrates an FPGA-based hardware implementation of this simulation, which reduces the computation time by 2-3 orders of magnitude. Thanks to this powerful tool, some significant improvements in the design of the coils have already been obtained.
\end{abstract}

\section{INTRODUCTION}

The preoccupation for improving the quality of life, for persons with different handicaps, led to extended research in the area of functional stimulation. Due to its advantages compared to electrical stimulation, magnetic stimulation of the human nervous system is now a common technique in modern medicine [1].

A difficulty of this technique is the lack of accurate focal stimulation. Another one is the low efficiency of power transfer from the coil to the tissue. To address these difficulties, coils with special geometries must be designed.

Because of the diversity of the medical applications involved, coils design requires testing a large number of geometries in order to find an adequate solution for the desired application [2], [4].

One of the major problems that appear in the design phase of such coils is the computation of the inductivity. For simple shapes of the coils (circular), one can determine analytical computation formulas [3], which are extremely complicated. When, however, the shape and the spatial distribution of the coil's turns do not belong to one of the known structures, a numerical method needs to be used for determining the inductivity of the coils.

The idea of the computation method is to divide the coils in small portions. Starting from this method, two computation systems are presented in the paper:

- The first one is classical and consists of a software implementation (Matlab);

- The second one consists of realizing a hardware architecture that exploits the intrinsic parallelism of the problem. The physical support of this architecture is an FPGA device.

The problem with the software implementation is its running time. Coils are designed by trial-and-error, and this approach is impractical if each trial requires half a day of computation. Besides, as this time grows with the complexity of the coil, it prevents designing complex coils. This paper shows that FPGA-based hardware acceleration is able to solve this bottleneck

The simulation of magnetic stimulators with complex forms requires dividing their coils in several parts. The self-inductance of the circuit, divided in $n$ parts, can be computed with formula (1). This mainly adds up the selfinductivities of the separate segments with the mutual inductivities of all the involved segments. The method is well described in [9]; the operations involved in computing the inductivity of a coil are: logarithm, division, addition and multiplication.

$$
L=\sum_{k=1}^{n} L_{k}+\sum_{k=1}^{n} \sum_{i=1}^{n} M_{k i}, \text { for }(i \neq k)
$$

The rest of the paper is organized as follows: Section 2 presents some considerations about the software algorithm. Section 3 deals with the hardware implementation and presents the global architecture as well as the architecture of the main building blocks. Section 4 makes a comparison 
between the two implementations, and Section 5 presents the main conclusions of this work.

\section{PRESENTATION OF THE ALGORITHM}

A coil is made up of a certain number of turns rolled around a central rod. Each turn can be considered as a perfect circle. The coil is structured on several vertical stages. The coil's parameters are: the outer radius, the wire's diameter and the distance (insulation) between consecutive turns

It is possible to have a different number of turns on every vertical stage. It is also possible to have a variable number of vertical stages, as shown in Fig. 1, where one can also notice the different number of turns on each stage.

A complex magnetic stimulation device contains a Slinky coil. Considering a coil with $N$ turns, the "Slinky-k" coil is generated by spatially locating these turns at successive angles of $i \times 180 /(k-1)$ degrees, were $i=0,1$, $\ldots, k-1$. If the current passing through this coil is $I$, then the central leg carries the total current $N \times I$. These coils are shown in Fig. 2, where each rectangle represents a leaf of the coil, viewed in perspective.

For computing the inductance, the turns are approximated by a finite number of points. We considered, after a series of tests, that a suitable amount of points on a turn is 64 . We have to take all points and combine them into segments made up of one point and the consecutive one. After this, each segment, one by one, is held as a reference. Then, the formula (1) is applied using this reference segment and all the other segments on the coil. For each pair of segments a value is obtained. These values must be added in order to obtain the coil's total inductance.

There are two phases for the functioning of the software implementation:

- In Phase 1 , the coordinates of the points are generated using trigonometric functions. They are also used in the hardware implementation.

- In Phase 2, the actual computation of the values is performed. Finally, we accumulate the values corresponding to the mutual and self inductivities.

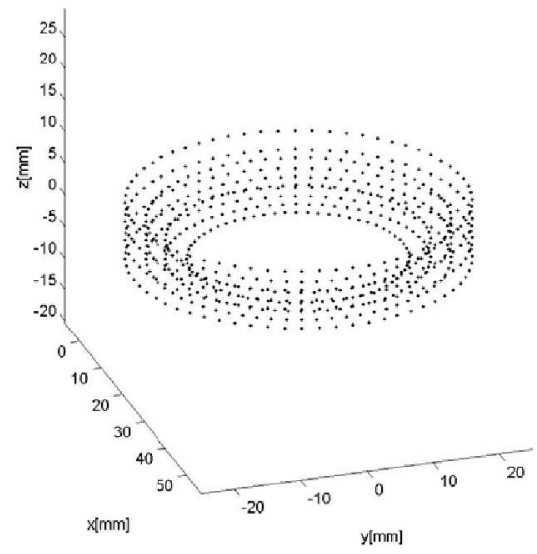

Fig. 1. Coil approximation using a finite number of points

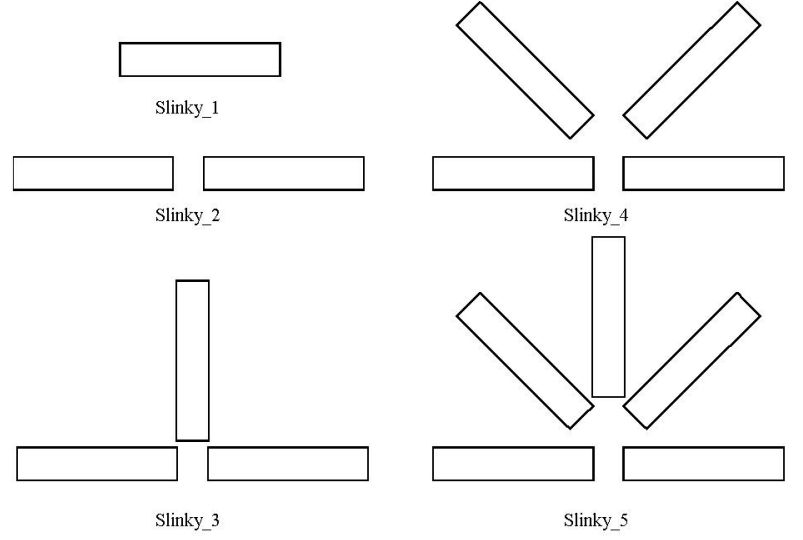

Fig. 2. Magnetic coil structures of the stimulation device

When we evaluate pairs of segments on the coil, three distinct cases can arise:

- The two segments are actually the same segment. In this case we add the segment's own self-inductivity. Since all segments have the same characteristics, this value is the same for all segments.

- The two segments are neighboring segments, that is, they have exactly one common point. Since the configuration is the same for all pairs of neighboring segments, this too is a constant value.

- The two segments are neither the same nor neighboring; they are two distinct segments in space. For complex configurations consisting of a large number of turns, this is the most general case, which accounts for most of the computation time.

The software implementation takes into account these considerations. We evaluate the third case by computing the mutual inductivities of all non-intersecting segments. The self-inductivity of the segments and the mutual inductivities of each segment against its neighboring ones are multiplied by the number of segments and accumulated at the end.

In order to evaluate the mutual inductivity of two separate segments in space we have introduced 5 variables denoted $v a r_{1}$ to $v a r_{5}$ which correspond to vector operations involving the two segments as described in the previous section. These variables will also be used in the next section. The points $\left(\mathrm{x}_{\mathrm{a}}, \mathrm{y}_{\mathrm{a}}, \mathrm{z}_{\mathrm{a}}\right)$ and $\left(\mathrm{x}_{\mathrm{b}}, \mathrm{y}_{\mathrm{b}}, \mathrm{z}_{\mathrm{b}}\right)$ correspond to the extremities of the first segment, while $\left(x_{c}, y_{c}, z_{c}\right)$ and $\left(x_{d}, y_{d}, z_{d}\right)$ correspond to the extremities of the second one.

$$
\begin{aligned}
\operatorname{var}_{1}= & \left(x_{b}-x_{a}\right) \cdot\left(x_{d}-x_{c}\right)+\left(y_{b}-y_{a}\right) \cdot\left(y_{d}-y_{c}\right)+\left(z_{b}-z_{a}\right) \cdot\left(z_{d}-z_{c}\right) \\
\operatorname{var}_{2}= & \sqrt{\left(x_{d}-x_{c}\right)^{2}+\left(y_{d}-y_{c}\right)^{2}+\left(z_{d}-z_{c}\right)^{2}} \\
\operatorname{var}_{3}= & \sqrt{\left(\left(x_{d}-x_{a}\right)+\left(x_{a}-x_{b}\right) \cdot t\right)^{2}+\left(\left(y_{d}-y_{a}\right)+\left(y_{a}-y_{b}\right) \cdot t\right)^{2}+\left(\left(z_{d}-z_{a}\right)+\left(z_{a}-z_{b}\right) \cdot t\right)^{2}} \\
\operatorname{var}_{4}= & \sqrt{\left(x_{a}+\left(x_{b}-x_{a}\right) \cdot t-x_{c}\right)^{2}+\left(y_{a}+\left(y_{b}-y_{a}\right) \cdot t-y_{c}\right)^{2}+\left(z_{a}+\left(z_{b}-z_{a}\right) \cdot t-z_{c}\right)^{2}} \\
\operatorname{var}_{5}= & \left(x_{d}-x_{c}\right) \cdot\left(x_{a}+\left(x_{b}-x_{a}\right) \cdot t-x_{c}\right)+\left(y_{d}-y_{c}\right) \cdot\left(y_{a}+\left(y_{b}-y_{a}\right) \cdot t-y_{c}\right)+ \\
& \left(z_{d}-z_{c}\right) \cdot\left(z_{a}+\left(z_{b}-z_{a}\right) \cdot t-z_{c}\right)
\end{aligned}
$$

These values are used for further computing the accumulation value: 
Accumulator $=$ Accumulator $+\frac{\operatorname{var}_{1}}{\operatorname{var}_{2}} \cdot \log \frac{\operatorname{var}_{3}+\operatorname{var}_{2}-\frac{\mathrm{var}_{5}}{\operatorname{var}_{2}}}{\operatorname{var}_{4}-\frac{\operatorname{var}_{5}}{\operatorname{var}_{2}}}$

The main drawback of a software implementation is the extremely high running time. It can be in the order of tens of minutes even for simple configurations, while for complex geometries of the coils it can exceed several hours (for instance, for a 58-turns coil, about 3 hours of computation time on a recent $\mathrm{PC}$ ).

Once the software simulation had been validated against actual coils, it was decided to try to accelerate it using custom hardware.

\section{HARDWARE IMPLEMENTATION}

\subsection{Floating point operators - FPLibrary}

Several libraries of floating-point operators for FPGAs have been published in the last few years. In this work, we use FPLibrary, developed at Ecole Normale Supérieure de Lyon and freely downloadable from [6]. Mantissa size and exponent size parameterize each operator in this library, allowing one to choose the precision and the dynamic range of the numbers.

The FPLibrary provides operators for addition, subtraction, multiplication, division and square root, some useful conversions and some elementary functions (currently exponential, logarithm and sine/cosine), in combinatorial or pipelined flavor. It is written in portable VHDL. It also offers operators for the alternative logarithmic number system [7].

The Core Generator tool, which comes with the Xilinx ISE, also offers floating-point operators. FPLibrary was chosen essentially because it offers a logarithm [8] which is not available in the Core Generator. However, it also proved more area-efficient. As our design requires a large number of operators in a tree-like pipeline, latency was not our main concern.

\subsection{System architecture}

The hardware implementation implies the same two phases as the software one, but Phase $l$ is not computationintensive and its implementation is kept in software.

In Fig. 3 a block diagram of the system is displayed. Three main blocks can be distinguished. The most important block is the pipeline stage, which receives values, computes them, and in a final stage accumulates them.

The coordinates are stored in a Block RAM memory There are 3 memories, one for each coordinate, $\mathrm{X}, \mathrm{Y}$, and $Z$. The synchronization logic, which gives the data to the pipeline, is implemented in a special interface. This interface consists of counters and latches.

The counters are orchestrated to generate the addresses

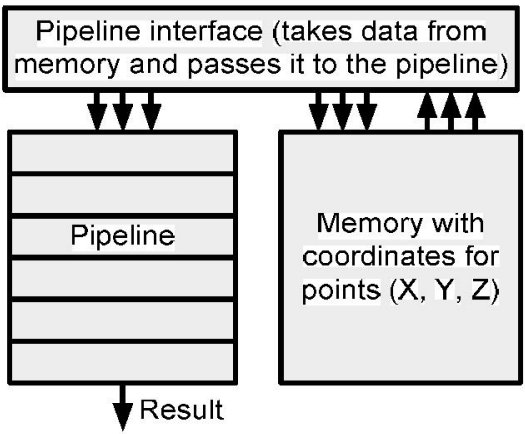

Fig. 3. Architecture of the hardware system

The latches are needed to implement a caching logic, which saves some of the memory used.

The pipeline stage consists of several sub-stages, based on the computations involved:

- A first stage computes the variables $v a r_{1}, v_{2}, v_{2}, r_{3}$, $v a r_{4}$ and $v a r_{5}$. There are several operations, which are common to the 5 variables. The pipeline reuses the corresponding intermediate values.

- The second stage computes the value to be accumulated. The latencies in this part of the pipeline are pretty large, up to 14 cycles, corresponding to waiting for a square root and about 29 cycles waiting for the logarithm and other operations to finish.

- The third stage is the accumulator. Because FPLibrary doesn't provide such a component one needed to be improvised using the existing resources. Special considerations were made in order to work around the specific latency that a simple adder introduces.

Not only the specific operators, but also buffer stages are used, which compensate the latency introduced by the operators. For instance, the addition operator introduces three cycles of latency, which must be compensated with three buffers. This also goes for the multiplication operator, which introduces four cycles of latency.

\subsection{Hardware implementation issues}

The performance and feasibility of the hardware implementation largely depends on its physical support. Our hardware platform was a Digilent Inc. board populated with a Xilinx Virtex2PRO30 FPGA device.

The problem was that this implementation is quite large: it depleted the space of the FPGA device we had available at that moment. To estimate the total space needed, we synthesized the design for a larger FPGA device (a Virtex4 $160 \mathrm{LX}$ ). A report of the device utilization is shown below:
Selected Device:

Number of Slices:

Number of Slice Flip Flops: Number of 4 input LUTs: 4vlx160ffl148-12

23656 out of $67584 \quad 35 \%$

20834 out of $135168 \quad 15 \%$ 44515 out of $135168 \quad 32 \%$ 


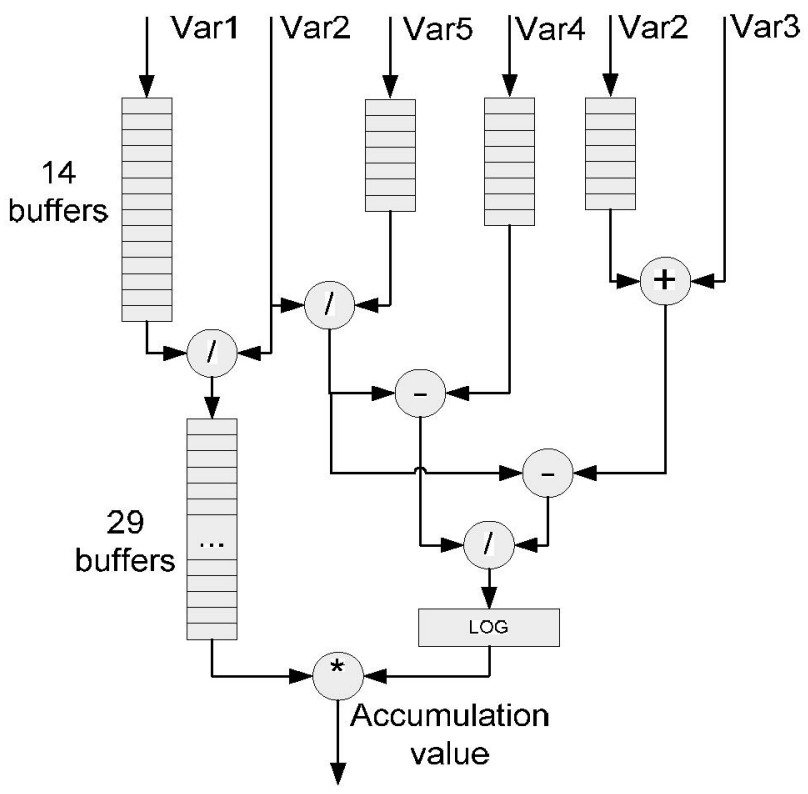

Fig. 4. Second stage: computing the accumulation value

The maximum frequency for this implementation was reported as $137.5 \mathrm{MHz}$. The implementation fits easily on this Virtex4 board. Regarding an implementation on our Virtex2Pro board two options were available.

The first option was to reduce the precision at which the pipeline operated. This ensured a reduction of both the buffer stages that provided the synchronization between the stages and a reduction in size of the operators. This option was first implemented. We reduced the mantissa of the operands by 10 bits. Instead of a large mantissa having 23 bits, the mantissa now had only 13 bits. Although the design fitted on a Virtex-II Pro board at about $98 \%$ of its capacity, the results obtained with this method were discouraging. They were more then $30 \%$ off from the actual result provided by Matlab. Therefore another method needed to be found.

The second option was to reduce the frequency at which the pipeline stage operates and time-multiplex some of the resources (square root - three occurrences in design, some of the adders). This has the advantage of preserving the pipeline's precision, the cost being a reduction in speed.
Selected Device:

Number of Slices:

2vp30ff896-6

Number of Slice Flip Flops:

Number of 4 input LUTs:

13380 out of 13696

15350 out of 27392

$97 \%$

$56 \%$ 24156 out of $27392 \quad 88 \%$

Of course we have a reduction in operating frequency: 85.714 MHz related to the weaker characteristics of the FPGA device and the more precise timing requirements.

\subsection{Improving the computation's precision}

It is known that in the process of accumulating large amounts of numbers, of very different exponents, a significant error can appear. This error is due to the fact that very small numbers, both positive and negative, have to be added to large numbers, and some of them are ignored (as shown in Fig. 5).

In order to eliminate those errors, a fixed-point giant accumulator was designed. The numbers which must be added are aligned with respect to the giant accumulator's fixed point (as shown in Fig. 6), and therefore there will be no losses if this accumulator's width is set to be large enough so that no numbers are shifted outside its range.

The giant accumulator is conceived to receive a floating point number which, according to its exponent, will be properly aligned for the accumulating step. The final result will be a fixed-point binary number, where the position of the radix point is previously set by the user.

Table 1 shows the results obtained from using these two types of accumulators, for some coils configurations. The configurations are given in the following format: number of turns per leaf, each leaf being structured on several vertical levels (for instance, configuration 7,6,6,6 $5,5-5,5-6,6,6,7$ has 25 turns on the first and fourth leaf, arranged on 4 levels; the first level has 7 horizontal turns, and the next ones 6 turns; the second and third leaves have 10 turns).

The results from the giant accumulator are more accurate, and therefore this one is more reliable in situations where a big precision is needed. For even more accurate results, one could set a larger width of the giant accumulator (currently this width is set to 64 bits).

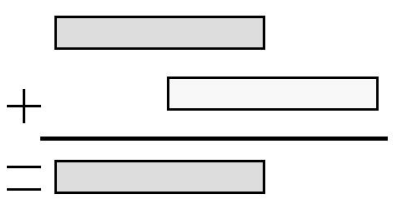

a)

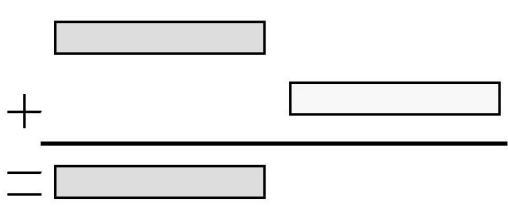

b)

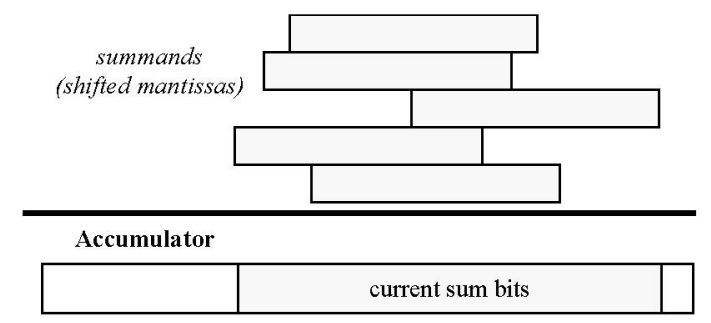

Fig. 6. The Giant Accumulator's principle

Fig. 5. Accumulation with very different exponents: a) Only part of the bits are discarded; b) All the bits are discarded 
Table 1. The values yielded by the two accumulators

\begin{tabular}{l|c|l|l} 
Nr. & Configuration & Classical FP Accumulator & Giant Accumulator \\
\hline \hline 1 & $5,5,5,5,5-5,5-5,5-5,5,5,5,5$ & 84.8 & 85.7 \\
\hline 2 & $5,5,5,5,5-4,3,3-3,3,4-5,5,5,5,5$ & 87.8 & 88.8 \\
\hline 3 & $7,6,6,6-5,5-5,5-6,6,6,7$ & 77.9 & 78.7 \\
\hline 4 & $7,6,6,6-4,3,3-3,3,4-6,6,6,7$ & 80.6 & 81.7 \\
\hline 5 & $8,7,5,5-5,5-5,5-5,5,7,8$ & 75.9 & 74.6 \\
\hline 6 & $8,7,5,5-4,3,3-3,3,4-5,5,7,8$ & 78.9 & 77.7 \\
\hline 7 & $8,7,6,4-5,5-5,5-4,6,7,8$ & 75.4 & 74.2 \\
\hline 8 & $8,7,6,4-4,3,3-3,3,4-4,6,7,8$ & 78.6 & 77.1 \\
\hline 9 & $8,7,7,3-5,5-5,5-3,7,7,8$ & 73.9 & 72.6 \\
\hline 10 & $8,7,7,3-4,3,3-3,3,4-3,7,7,8$ & 76.9 & 75.5 \\
\hline
\end{tabular}

\section{EXPERIMENTAL RESULTS}

\subsection{Validation of the hardware architecture}

The main achievement of the hardware implementation over the software one is the reduction in computation time. By performing one accumulation per clock cycle the hardware solution is indeed efficient and can be used even for the most complex magnetic stimulation systems.

In terms of complexity, both implementations, in software and in hardware, have the same complexity, $O\left(n^{2}\right)$ with $n$ being the number of distinct segments. The hardware structure performs one accumulation per clock cycle, which means that each clock cycle a mutual inductivity between two segments is evaluated. The software implementation performs the same computation in a longer time.

First we analyzed simpler cases, 1 to 4 turns. The outer turns are the widest turns on the coil while the inner turns are the neighbors of the outer turns located closer to the center. Then the results for the given configurations are presented. The analyzed quantity was the inductivity.

We analyzed our software and hardware implementations using three distinct configurations (Fig. 7). The values produced by the implementations are given in Table 2, where one can see the comparison between the results provided by the software and the hardware solutions.

These preliminary results showed that the hardware implementation was, for practical purposes, as accurate as the software one, and was several orders of magnitude faster. We therefore could use it to simulate more complex configurations which are out of reach of the software version, such as those required to obtain the $30 \mu \mathrm{H}$ advocated by recent references in this field [2] (necessary for an improved efficiency of the energy transfer from the coil to the target tissue).

\subsection{Using the hardware implementation for actual simulations}

The tested coils only had 18 turns, because larger structures could not be efficiently analyzed using the software implementation. Therefore, based on the inductivity calculus described here and performed with a less timeconsuming technique - the hardware implementation, we were able to estimate the inductivity of 22 different configuration of a Slinky_4 coil with 70 turns. The coil has an outer radius of each leaf equal to $30 \mathrm{~mm}$, an insulation gap between turns of $0.2 \mathrm{~mm}$ and the wire radius is $1 \mathrm{~mm}$.

This estimation allows further investigations regarding the importance of coil configuration and geometry on the focality and efficiency of the stimulation (of course, the hardware technique implemented can be applied to the inductivity computation of any other structure of coil.)

Table 3 contains the values of the inductivity computed for the given coils configurations (the number of turns per leaf, each leaf being structured on several vertical levels). The first 10 values have already been presented in Table 1.

These values are now already in use for the estimation of energetic parameters of stimulation coils and results are to be published in our future works. But it is now important to emphasize the fact that performing a software computation of the inductivities of such a large number of different coils (each one divided in 4,480 segments) would have been practically extremely difficult to perform.

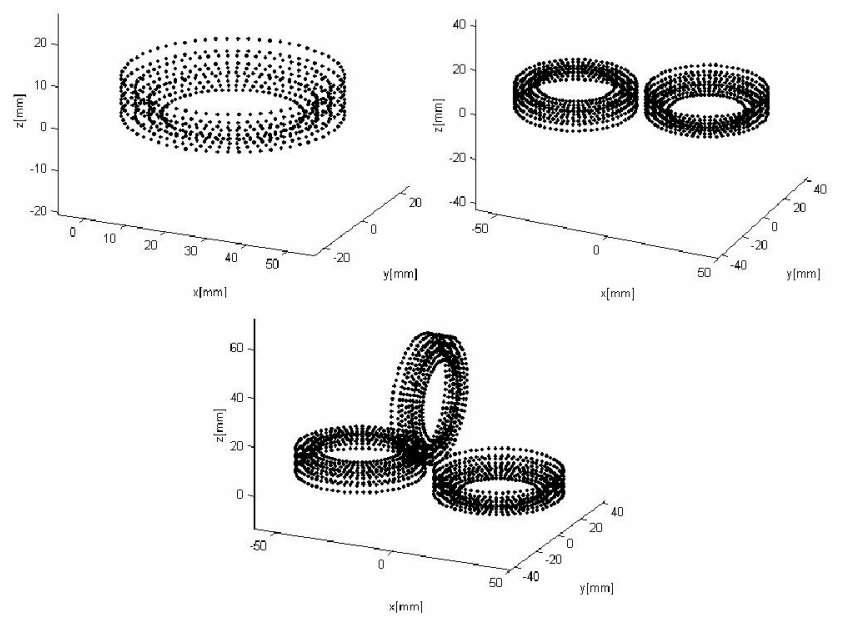

Fig. 7. Analyzed configurations 
Table 2. Comparison of results

\begin{tabular}{|c|c|c|c|c|c|}
\hline Configuration & $\begin{array}{l}\text { Inductivity } \\
\text { (hardware) } \\
{[\mu \mathrm{H}]}\end{array}$ & $\begin{array}{l}\text { Inductivity } \\
\text { (software) } \\
{[\mu \mathrm{H}]}\end{array}$ & $\begin{array}{l}\text { Duration } \\
\text { (hardware) } \\
\text { [no. of clock cycles] }\end{array}$ & $\begin{array}{c}\text { Running speed } \\
\text { (hardware - at } 85.71 \\
\mathrm{MHz} \text { ) [seconds] }\end{array}$ & $\begin{array}{c}\text { Running speed } \\
\text { (software) } \\
\text { [seconds] } \\
\end{array}$ \\
\hline "Slinky_1 coil ( 1 outer turn) & 0.097 & 0.097 & 40,960 & 0.00047 & $\overline{4.2}$ \\
\hline Slinky_1 coil ( 2 outer turns) & 0.30 & 0.30 & 163,840 & 0.00194 & 18 \\
\hline Slinky_ 1 coil 4 turns ( 2 out. 2 in.) & 0.92 & 0.93 & 655,360 & 0.00764 & 72 \\
\hline Slinky_1 coil (10 turns) & 3.81 & 3.9 & $4,096,000$ & 0.04705 & 420 \\
\hline Slinky_2 coil (10-10) & 8.4 & 8.6 & $16,384,000$ & 0.19411 & 1,680 \\
\hline Slinky_3 coil $(10-10-10)$ & 13.32 & 13.6 & $36,864,000$ & 0.42941 & 3,600 \\
\hline
\end{tabular}

Table 3. Inductivities of different coils configurations \begin{tabular}{l}
$\mathrm{Nr}$. Inductivity L $[\mu \mathrm{H}] \mid \quad$ Configuration \\
\hline \hline
\end{tabular}

\begin{tabular}{c|c|c}
\hline \hline 1 & 75.0 & $9,8,5,4-5,5-5,5-4,5,8,9$ \\
\hline 2 & 78.0 & $9,8,5,4-4,3,3-3,3,4-4,5,8,9$ \\
\hline 3 & 72.2 & $10,5,5,5-5,5-5,5-5,5,5,10$ \\
\hline 4 & 75.1 & $10,5,5,5-4,3,3-3,3,4-5,5,5,10$ \\
\hline 5 & 70.1 & $10,6,6,3-5,5-5,5-3,6,6,10$ \\
\hline 6 & 73.1 & $10,6,6,3-4,3,3-3,3,4-3,6,6,10$ \\
\hline 7 & 63.6 & $11,8,6-5,5-5,5-6,8,11$ \\
\hline 8 & 66.3 & $11,8,6-4,3,3-3,3,4-6,8,11$ \\
\hline 9 & 64.6 & $10,8,7-5,5-5,5-7,8,10$ \\
\hline 10 & 64.1 & $10,8,7-6,4-6,4-7,8,10$ \\
\hline 11 & 63.9 & $10,8,7-4,3,3-3,3,4-7,8,10$ \\
\hline 12 & 65.8 & $9,8,8-5,5-5,5-8,8,9$ \\
\hline
\end{tabular}

\section{CONCLUSIONS AND FUTURE WORK}

The equipment used in magnetic stimulation of the nervous system is costly and bulky. This is mainly due to the fact that the currents flowing through the stimulation coil are very intense $(\mathrm{kA})$, leading to coil heating and strong electromagnetic forces that might destroy the coil. Therefore, magnetic coil design is one of the most important aspects of the technique of magnetically stimulating the nervous system.

Since every medical application requires its own optimal structure of the magnetic coil, the results emphasized in this paper can play an important role for future work on coil design.

Because of the large amount of operations involved (several tens of millions just for one coil) it is very hard to debug such a hardware system at least at an acceptable level, but the obtained results show an excellent concordance with those obtained in software. Our implementation has the advantage of greatly speeding up the computation time and hence shortening the design process. On larger FPGA devices the process can achieve a greater speed by accommodating more computational structures in parallel. Besides, on larger devices, we will also have the possibility of increasing the precision of the floating-point format to use this architecture on larger coils.
The development of an optimized norm operator (to be included in FPLibrary) will provide a space efficient alternative to the combination of multipliers and adders we currently use in the implementation and would probably enhance the latency as well.

The powerful FPGA-based computational tool described in this paper allows us to compute both the coil's inductivity and the magnetic field's value on a given point in a short time (a few minutes, which is much less than the time required by a software run -3 hours for a 58-turns coil, and the runtime grows quadratically).

\section{REFERENCES}

[1] D. Mozeg and E. Flak. "An Introduction to Transcranial Magnetic Stimulation and Its Use in the Investigation and Treatment of Depression," University of Toronto Medical Journal, vol. 76, no.3, 1999, pp. 158-162.

[2] I. Griškoval, and J. Höppner, "Transcranial magnetic stimulation: the method and application," Medicina (Kaunas) 2006; 42(10), pp. 792-804

[3] K. S. Han. "Self-inductance of air-core circular coils with rectangular cross section," IEEE Transactions on Magnetics, vol. 23, no. 6, November 1987, pp. 3916- 3921.

[4] L Creț and R. Ciupa, "Remarks on the Optimal Design of Coils for Magnetic Stimulation," ISEM Proceedings, Bad Gastein, Austria., 2005, pp. 352-354.

[5] P. Kalantarov and L. Teitlin, "Calculul inductivităţilor”, Ed. Tehnică, Bucuresti, 1958.

[6] http://www.ens-lyon.fr/LIP/Arenaire/Ware/FPLibrary/

[7] J. Detrey and F. de Dinechin, "A tool for unbiased comparison between logarithmic and floating-point arithmetic", Journal of VLSI Signal Processing, vol. 49, no. 1, Springer, 2007, 161-175

[8] J. Detrey, F. de Dinechin. "A Parameterizable Floating-Point Logarithm Operator for FPGAs," in Proceedings of the 39th Asilomar Conference on Signals, Systems \& Computers, November 2005, pp. $1186-1190$.

[9] L. Creț, M. Pleșa, D. D. Micu and R. Ciupa. "Magnetic Coils Design for Focal Stimulation of the Nervous System", in Proceedings of EUROCON 2007, 9-12 September 2007, Warsaw, Poland, pp. 1998-2003. 